\title{
Radiographic and clinical outcomes using intraoperative magnetic resonance imaging for transsphenoidal resection of pituitary adenomas
}

\author{
Rupa G. Juthani, MD, ${ }^{1,2}$ Anne S. Reiner, MPH, ${ }^{3}$ Ankur R. Patel, MD, ${ }^{2}$ Aimee Cowan, BA, ${ }^{2}$ \\ Marie Roguski, MD, MPH, ${ }^{2,4}$ Katherine S. Panageas, DrPH, ${ }^{3}$ Eliza B. Geer, MD, ${ }^{5,8}$ \\ Sasan Karimi, MD, ${ }^{6}$ Marc A. Cohen, MD, MPH, ${ }^{7,8}$ and Viviane Tabar, MD ${ }^{2,8}$
}

\begin{abstract}
${ }^{1}$ Department of Neurosurgery, Weill Cornell Medical College, New York; Departments of ${ }^{2}$ Neurosurgery, ${ }^{3}$ Epidemiology and Biostatistics, ${ }^{5}$ Medicine, ${ }^{6}$ Radiology, and ${ }^{7} \mathrm{Head}$ and Neck Surgery, Memorial Sloan Kettering Cancer Center, New York, New York; ${ }^{4}$ Department of Neurosurgery, Tufts University, Boston, Massachusetts; and ${ }^{8}$ Multidisciplinary Pituitary and Skull Base Tumor Center, Memorial Sloan Kettering Cancer Center, New York, New York
\end{abstract}

\begin{abstract}
OBJECTIVE The utility and safety of intraoperative MRI (iMRI) for resection of pituitary adenomas is not clearly established in the context of advances in endoscopic approaches. The goal in this study was to evaluate the safety and efficacy of iMRI for pituitary adenoma resection, with endoscopic transsphenoidal (ETS) versus microscopic transsphenoidal (MTS) approaches.
\end{abstract}

METHODS Radiographic and clinical outcomes of all pituitary adenomas resected using iMRI between 2008 and 2017 at a single institution were retrospectively evaluated.

RESULTS Of 212 tumors treated, 131 (62\%) underwent further resection based on iMRI findings, resulting in a significant increase in gross-total resection on postoperative MRI compared with iMRI $(p=0.0001)$ in both ETS and MTS groups. iMRI increased rates of gross-total resection for cavernous sinus invasion Knosp grades 1 and 2, but not in Knosp $\geq 3$ across treatment groups ( $p$ 0.0001). The extent of resection on postoperative MRI was significantly correlated with increased progression-free survival $(p<0.0001)$. Initial hormone remission off medical therapy was achieved in $64 \%$, with a significantly higher rate of remission in tumors resected via the ETS approach (81\%) compared with the MTS approach $(55 \%)(p=0.02)$. The rate of persistent new hormone deficit was low at $8 \%$, including a $2.8 \%$ rate of permanent diabetes insipidus, and $45 \%$ of patients had improvement in preoperative hormone deficit following surgery. Serious postoperative complications including CSF leaks requiring reoperation were rare at 1\%, with no postoperative infections.

CONCLUSIONS These results suggest that iMRI is a safe and effective method of increasing the extent of resection for pituitary adenomas while preserving hormone function. When paired with the endoscope, iMRI may offer the ability to tailor more aggressive removal of tumors while optimizing pituitary function, resulting in high rates of secretory hormone remission. Secretory tumors and adenomas with Knosp grade $<3$ cavernous sinus invasion may benefit most from the use of iMRI.

https://thejns.org/doi/abs/10.3171/2020.4.JNS20178

KEYWORDS pituitary adenoma; transsphenoidal; intraoperative MRI; pituitary endocrine outcomes; pituitary extent of resection; secretory adenoma; pituitary surgery

$\mathrm{E}$ NDOSCOPIC transsphenoidal (ETS) approaches to the sella turcica have transformed the surgical landscape of pituitary tumors, allowing for more extensive resections due to enhanced visualization, with improvement in nasal outcomes ${ }^{1-3}$ and potentially lower rates of new hormone deficits, compared with microscopic trans- sphenoidal (MTS) surgery. ${ }^{1,4}$ Nonetheless, there remains a high percentage of patients with pituitary adenomas who have residual or recurrent disease, with gross-total resection (GTR) rates as low as 25\%-40\% for larger tumors, and common residual disease even with small tumors. Surgeries for recurrent/residual disease are often compli-

ABBREVIATIONS ACTH = adrenocorticotropic hormone; $\mathrm{CSI}=$ cavernous sinus invasion; $\mathrm{DI}=$ diabetes insipidus; EOR = extent of resection; ETS = endoscopic transsphenoidal; GH = growth hormone; GTR = gross-total resection; iMRI = intraoperative MRI; MTS = microscopic transsphenoidal; NFA = nonfunctioning adenoma; NTR = near-total resection; PFS = progression-free survival; PRL = prolactin; RFS = recurrence-free survival; STR = subtotal resection; VF = visual field.

SUBMITTED January 17, 2020. ACCEPTED April 10, 2020.

INCLUDE WHEN CITING Published online July 3, 2020; DOI: 10.3171/2020.4.JNS20178. 
cated by difficulty preserving normal structures, resulting in higher rates of subtotal resection (STR) $)^{5,7}$ and new hormone deficits. ${ }^{7}$ In secretory tumors, particularly with adrenocorticotropic hormone (ACTH), even small residual tumors can be associated with high rates of morbidity. ${ }^{8}$ Although reported resection rates are generally higher in patients treated endoscopically compared with microscopically, ${ }^{5,9}$ concern has been raised regarding the potential increased risk of endocrine deficits and CSF leaks.

The use of intraoperative MRI (iMRI) has been rapidly adopted to increase the extent of resection (EOR) for brain tumors, particularly for gliomas. ${ }^{10,11}$ Its use for benign tumors is more poorly defined, but there is growing interest in using iMRI for resection of pituitary adenomas. The sensitivity and specificity of iMRI in detecting residual pituitary adenoma have been reported to be nearly $100 \%$, in comparison with the endoscope, which is reported to have a sensitivity of $21 \%$ and specificity of $78 \% .^{34}$ The use of iMRI has been reported to increase $\mathrm{EOR}^{6,12}$ by $15 \%-83 \%$. In a recent review ${ }^{13}$ of 85 studies reporting the use of iMRI for pituitary adenomas, the authors found higher GTR rates in the endoscope plus iMRI group, but they were unable to draw direct comparisons due to series heterogeneity, with limited hormone outcome data. Recent pertinent series are compared in Table 1.,13-22 Overall, studies support a potential role for iMRI in increasing both EOR and progression-free survival (PFS) for pituitary adenomas, but lack clarity on the magnitude of improvement, particularly in terms of endocrine outcomes. ${ }^{23}$

This study aimed to evaluate the role of iMRI in impacting EOR and PFS, with a detailed analysis of secretory hormone outcomes and endocrine function following resection. Microscopic and endoscopic outcomes are compared to evaluate the optimal use of iMRI paired with one or both methods.

\section{Methods}

All pituitary adenomas resected by a single neurosurgeon with the aid of iMRI between 2008 and 2017 were retrospectively reviewed with the use of a prospectively constructed database and after IRB approval and waiving of consent. During this period of time, all pituitary adenomas were resected using iMRI except in rare (11 total) cases in which iMRI was not used for technical or clinical reasons, including the lack of availability of iMRI and contrast allergy. All patients underwent preoperative pituitary hormone-level testing, visual field (VF) examination, and MRI prior to surgery. Dedicated pituitary protocol MR images were used for dimensions, volumetric analysis, evaluation of optic chiasm displacement, suprasellar involvement, and cavernous sinus invasion (CSI) as described in Knosp et al. ${ }^{33}$ All patients had documentation of preoperative hormone status, including preoperative hormone deficits and presence of hormone secretion. An MTS approach was performed prior to 2014 in a total of 130 cases. After 2014, all cases were performed using the endoscope, including 75 tumor resections performed with the endoscope alone. Seven tumors were resected using a combined approach during the transition period from 2013 to 2014.

\section{Operating Room Setup and Imaging}

All patients underwent surgery in a BrainSuite-equipped operating room, consisting of a fixed intraoperative 1.5-T MRI scanner (Siemens AG) integrated with neuronavigation (Brainlab). EOR was evaluated based on analysis of thin-slice (2-mm) coronal T2-weighted and 3D contrastenhanced T1-weighted images by an independent neuroradiologist and documented in real time during surgery. The EOR was categorized as GTR, near-total resection (NTR; defined as $<10 \%$ residual volume), or STR. Postoperative EOR was evaluated based on initial postoperative MRI studies obtained prior to further treatment, usually performed within 3-6 months, and was categorized as GTR, NTR, STR, or recurrence/growth. The last available MRI study was evaluated for assessment of final tumor control at last follow-up. Brainlab imaging software was used for volumetrics. Postoperative imaging was available in 200 of the 212 patients.

\section{Surgical Procedure}

Patients were positioned supine in a slightly extended midline position in a fixed MRI-compatible frame intended for iMRI. All patients underwent a transsphenoidal approach using frameless stereotactic navigation. A standard MTS or ETS approach was used to gain access to the sella. All ETS cases were performed in collaboration with a single ENT surgeon, whereas purely MTS cases were typically performed by the neurosurgeon alone. Once the surgeons estimated that a maximal safe resection was achieved, including cases in which residual tumor was thought to be inaccessible or indistinguishable from normal gland, iMRI was performed following Gd administration. Additional resection was performed in cases of accessible residual tumor on iMRI. In the ETS approach, $30^{\circ}$ and $45^{\circ}$ angled endoscopes were used to evaluate the surgical bed and extend the resection where indicated. Repair of CSF leaks was performed using a range of methods, including Gelfoam, sealants, dural substitutes, and nasoseptal flaps alone or in combination.

\section{Endocrine Evaluation}

All patients underwent preoperative hormone testing for $\mathrm{ACTH} / \mathrm{cortisol}$, luteinizing hormone/follicle-stimulating hormone $(\mathrm{LH} / \mathrm{FSH}) \pm$ testosterone, thyroid-stimulating hormone/thyroxine (TSH/T4), growth hormone/ insulin-like growth factor-1 (GH/IGF-1), and prolactin (PRL) to determine hyper- or hyposecretion, based on normal references for each hormone. Patients were evaluated by an endocrinologist in a majority of cases and in all secretory cases. PRL hypersecretion was determined to be attributable to stalk effect rather than primary secretory tumor when the value was $<100 \mathrm{ng} / \mathrm{mL}$ in the appropriate clinical setting (i.e., macroadenoma). Elective resection of prolactinomas was limited to patients whose disease was refractory to or who were intolerant of medical management due to side effects or fertility status.

Initial postoperative hormone remission was defined as normalization of serum values of secretory hormones off medication without further treatment. Final hormone remission was evaluated at last follow-up following treat- 


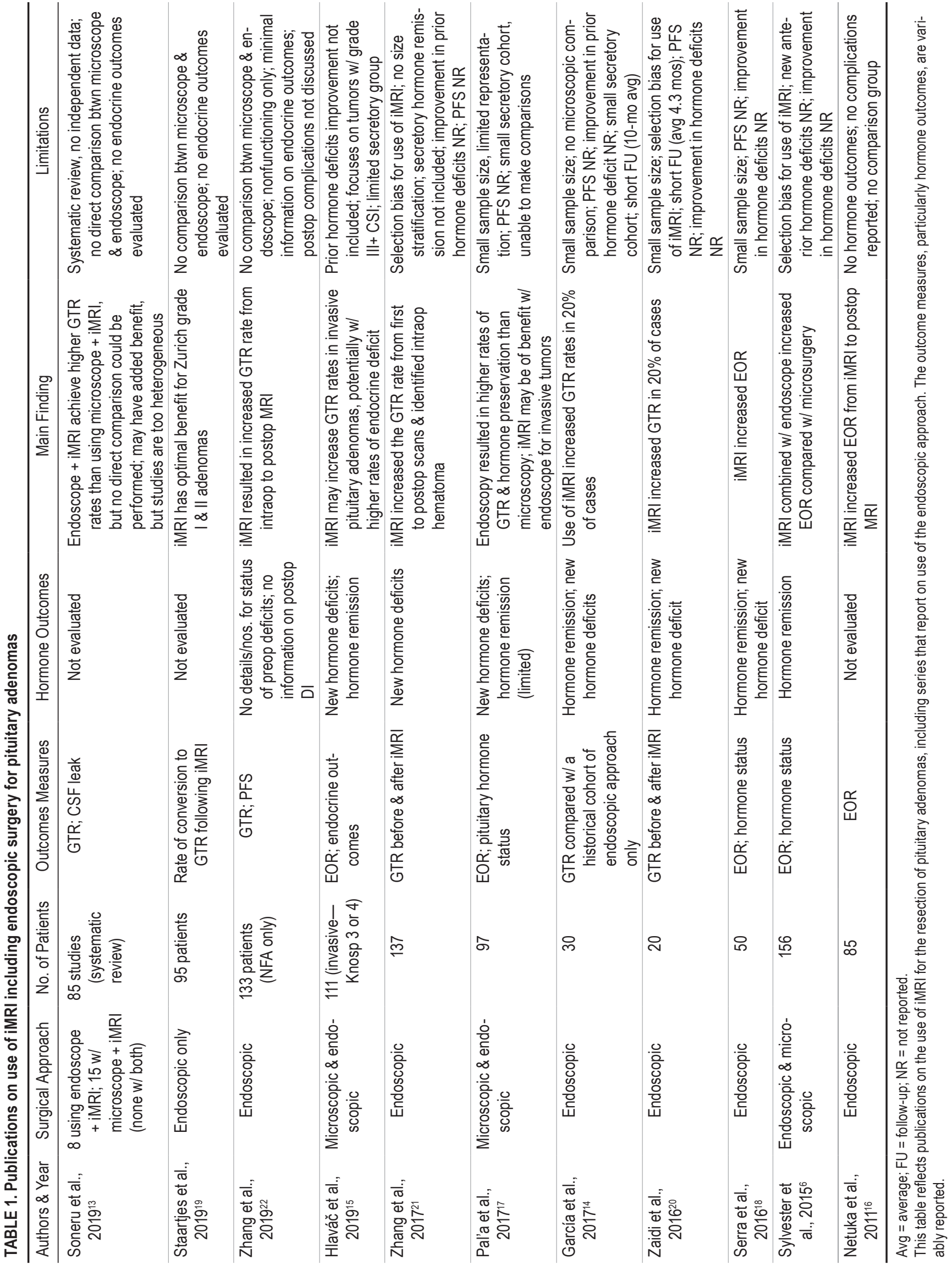


ment for residual or recurrent hormone secretion, including medication.

\section{Statistical Analysis}

Chi-square tests and Fisher's exact tests, where appropriate, were used to compare categorical variables by groups of interest. The Wilcoxon 2-sample test was used to compare numerical variables by 2 groups of interest. The Kruskal-Wallis test was used to compare numerical variables by more than 2 groups of interest. McNemar's test was used to quantify movement in EOR (NTR/STR to GTR) from iMRI to initial postoperative MRI. Radiographic PFS was calculated from the time of surgery until radiographic progression, death, or last follow-up, whichever occurred first. Secretory recurrence survival was calculated from the time of surgery until secretory progression, death, or last follow-up, whichever occurred first. Kaplan-Meier methodology was used for survival analyses and curves were compared using the log-rank test. All tests were 2-sided with a level of statistical significance less than 0.05. Statistical analyses were performed and figures were generated in SAS (version 9.4, SAS Institute) and R 3.5.2 using the ggplot2 and survival packages.

\section{Results \\ Patient Demographics and Preoperative Tumor Characteristics}

A total of 212 adenomas for which clinical and radiographic follow-up was available were included, including 130 cases performed via MTS, 75 via ETS, and 7 via a combination of these approaches (Table 2). Preoperative variables across groups demonstrated no statistically significant differences (Table 3). This series included $33 \mathrm{mi}-$ croadenomas $(16 \%)<1 \mathrm{~cm}$ in size, including 1 case of "MRI-negative" Cushing's disease, with the majority of tumors representing macroadenomas. The mean tumor volume was $4.38 \mathrm{~cm}^{3}$, with a majority demonstrating optic chiasm displacement (59\%) and suprasellar extension (72\%). CSI was present in 110 cases (52\%), with $11 \%$ showing Knosp $\geq 3$ CSI. Of the 212 adenomas, $34 \%$ were secretory and 66\% were nonfunctioning adenomas (NFAs). Twenty-nine of 72 (40\%) secretory tumors were GH-secreting, 20 (28\%) were corticotropin (ACTH)-secreting, and 23 (32\%) were PRL-secreting tumors. Of the 23 patients with PRL-secreting tumors that underwent resection, 17 (74\%) were intolerant of or refractory to medical therapy, 3 (13\%) refused surgery due to fertility concerns, 1 (4\%) had radiographic features of craniopharyngioma, 1 (4\%) had evidence of apoplexy with clinical need for Avastin treatment, and 1 (4\%) was thought to have an abnormally elevated stalk effect. Thirty percent of patients had one or more hormone deficits preoperatively. These results are summarized in Table 3.

\section{Radiographic Outcomes}

A total of 131/212 (62\%) patients underwent further resection based on iMRI findings. This included 47/75 (63\%) ETS and 79/130 (61\%) MTS cases undergoing further resection (Table 4). The rate of GTR was significantly increased after further resection across all treatment groups
TABLE 2. Demographic data in patients with pituitary adenomas

\begin{tabular}{lc}
\hline \multicolumn{1}{c}{ Characteristic } & Value \\
\hline Total patients, no. (\%) & 212 \\
\hline Microscopic resection & $130(61)$ \\
\hline Endoscopic resection & $75(35)$ \\
\hline Combination & $7(3)$ \\
\hline Age in yrs, mean (range) & $51.0(16.1-83.0)$ \\
\hline Sex, no. (\%) & $89(42)$ \\
\hline Male & $123(58)$ \\
\hline Female & $196.3(74.0-490.0)$ \\
\hline Duration of procedure in mins, mean (range) & $158.95(74-322)$ \\
\hline Microscopic resection & $252.96(146-490)$ \\
\hline Endoscopic resection & $283.29(211-466)$ \\
\hline Combination & $3.6(0.0-13.0)$ \\
\hline Length of stay in days, mean (range) & $32.7(0.3-125.6)$ \\
\hline Clinical FU in mos, mean (range) & $42.5(0.36-125.6)$ \\
\hline Microscopic resection & $17.3(0.49-55.4)$ \\
\hline Endoscopic resection & $16.9(0.3-53.3)$ \\
\hline Combination & $33.3(0-118.9)$ \\
\hline Radiographic FU in mos, mean (range) & $42.6(0-118.9)$ \\
\hline Microscopic resection & $17.3(0.07-55.9)$ \\
\hline Endoscopic resection & $35.0(6.4-58.8)$ \\
\hline Combination & \\
\hline Patient demographic data, surgical times, overall length of stay, and follow-up \\
times are reported. \\
${ }^{*}$ This includes 200 total patients (12 lost to radiographic follow-up).
\end{tabular}

(McNemar's $\mathrm{p}=0.0001)$, with initial postoperative MRI consistent with GTR in 105 cases (53\%), NTR in 55 cases (28\%), and STR in 39 cases (20\%), and 1 early recurrence. For patients who did not achieve GTR, further resection following iMRI resulted in improvement from STR to NTR in 16 cases (13\%; Fig. 1). Notably, the NTR group may include a small number of cases in which residual disease was indistinguishable from postoperative changes, necessitating further follow-up on subsequent MR images.

Comparing the MTS and the ETS group, rates of resection on initial postoperative MRI were significantly higher in the ETS group $(\mathrm{p}=0.00056)$, where GTR was achieved in $69 \%$ of cases (50/72) compared with $44 \%$ (54/123) in the MTS group. Compared with iMRI, an additional $37 \%$ of patients converted to GTR on initial postoperative MRI (23\% in the MTS and 64\% in the ETS group; $p=0.0001$ ).

Radiographic progression occurred in $15 \%$, with a total of 10 cases (7\%) requiring further surgery (Table 4). This excludes a total of 12/212 cases for which postoperative radiographic follow-up was not available. Rates of GTR were significantly higher in the ETS group compared with the MTS group at both initial postoperative MRI $(\mathrm{p}=0.0006)$ and final postoperative MRI $(\mathrm{p}=0.0007)$, although GTR rates on iMRI were similar. However, the difference in follow-up time in the MTS group (42.6 months) was significantly higher than in the ETS group (17.3 months), limiting meaningful comparison of recurrence rates between these 2 cohorts.

EOR was strongly correlated with increased PFS on 
TABLE 3. Preoperative patient and tumor characteristics

\begin{tabular}{|c|c|c|c|}
\hline Characteristic & $\begin{array}{c}\text { Total Cohort, } \\
n=212^{*}\end{array}$ & $\begin{array}{l}\text { Microscopic Only, } \\
n=130\end{array}$ & $\begin{array}{l}\text { Endoscopic Only, } \\
\qquad n=75\end{array}$ \\
\hline Secretory & $72(34)$ & $42(32)$ & $27(36)$ \\
\hline $\mathrm{GH}$ & $29(40)$ & $17(40)$ & $11(41)$ \\
\hline $\mathrm{ACTH}$ & $20(28)$ & $9(21)$ & $10(37)$ \\
\hline PRL & $23(32)$ & $16(38)$ & $6(22)$ \\
\hline NFA & $140(66)$ & $88(68)$ & $48(64)$ \\
\hline \multicolumn{4}{|l|}{ Radiographic characteristics } \\
\hline Optic chiasm displacement & $125(59)$ & $76(58)$ & $43(57)$ \\
\hline Suprasellar involvement & $153(72)$ & $93(72)$ & $55(73)$ \\
\hline CSI & $110(52)$ & $66(51)$ & $38(51)$ \\
\hline Knosp $\geq 3$ & $24(11)$ & $14(11)$ & $7(9)$ \\
\hline \multicolumn{4}{|l|}{ Surgical history } \\
\hline No prior & $181(85)$ & $112(86)$ & $64(85)$ \\
\hline Revision & $31(15)$ & $18(14)$ & $11(15)$ \\
\hline \multicolumn{4}{|l|}{ Hormone deficit } \\
\hline None & $148(70)$ & $96(74)$ & $49(65)$ \\
\hline$\geq 1$ & $64(30)$ & $34(26)$ & $26(35)$ \\
\hline VF deficit & $81(38)$ & $53(41)$ & $25(33)$ \\
\hline Tumor vol in $\mathrm{cm}^{3}$, mean (range) & $4.38(0.01-35.16)$ & $4.45(0.01-35.2)$ & $3.89(0.01-31.02)$ \\
\hline \multicolumn{4}{|l|}{ Tumor size, max diam } \\
\hline MRI negative & $1(0.5)$ & $1(0.8)$ & $0(0)$ \\
\hline$<1 \mathrm{~cm}$ & $32(15)$ & $20(15)$ & $12(16)$ \\
\hline $1-2 \mathrm{~cm}$ & $63(30)$ & $40(31)$ & $23(31)$ \\
\hline$>2-3 \mathrm{~cm}$ & $85(40)$ & $47(36)$ & $35(47)$ \\
\hline$>3 \mathrm{~cm}$ & $31(15)$ & $22(17)$ & $5(7)$ \\
\hline \multicolumn{4}{|c|}{$\begin{array}{l}\text { Max diam = maximum diameter. } \\
\text { Preoperative tumor characteristics are presented based on surgical approach. There were no significant differences in } \\
\text { preoperative characteristics between the groups. Unless otherwise indicated, values are expressed as the number of } \\
\text { patients (\%). } \\
{ }^{*} \text { This includes } 7 \text { patients treated via a combined MTS and ETS approach, with totals reflective of all categories. }\end{array}$} \\
\hline
\end{tabular}

initial postoperative MRI ( $\mathrm{p}<0.0001$; Fig. 2A). Radiographic PFS did not differ between the ETS (not reached) and MTS (107.4 months, 95\% CI 98.96 months-no upper limit; $p=0.78$ ) groups (Fig. 2B), including subgroup analysis for NFAs ( $\mathrm{p}=0.77$; Fig. $2 \mathrm{C})$ and secretory adenomas $(\mathrm{p}=0.92$; Fig. 2C).

Radiographic outcomes were further evaluated as a function of CSI by Knosp grading (Table 5). For CSI $\geq$ 3 , there was no increase in GTR rates following further resection, with the exception of 1 case read as GTR on iMRI but suspicious for residual on initial postoperative MRI. In the Knosp < 3 CSI (grades 1 and 2) subgroup, GTR rates were significantly increased following further resection after iMRI ( $\mathrm{p}<0.0001)$, particularly with ETS, resulting in a GTR rate of $70 \%$ in the endoscopic group but only $22 \%$ in the microscopic group. In cases with no CSI, there was more parity between the MTS and ETS groups, with rates of GTR on postoperative MRI at $72 \%$ and $80 \%$, respectively $(\mathrm{p}=0.37)$.

\section{Hormone Outcomes}

For secretory tumors, further resection after iMRI resulted in 50\% of cases achieving GTR, with an additional 18\% increasing from STR to NTR. This resulted in a significant increase in GTR for secretory tumors $(\mathrm{p}=$ 0.0008 ) across treatment groups. Initial hormone remission off medical therapy was achieved in $64 \%$ of cases (66\% of GH-secreting, $80 \%$ of ACTH-secreting, and $48 \%$ of PRL-secreting tumors), with $81 \%$ achieving remission in the ETS versus 55\% in the MTS group $(\mathrm{p}=0.02)$. The median secretory recurrence-free survival (RFS) was 81.6 months (95\% CI 47.6-100.2 months) in the total cohort but was not reached in the ETS group (Table 6, Fig. 2D).

Treatment for persistent hormone secretion was required in a total of 35/72 (49\%) cases, including 33\% of ETS and $57 \%$ of MTS cases. The majority of cases (23/35, $66 \%$ were controlled with medication alone. At last follow-up after subsequent treatment, hormone control was achieved in $75 \%$ of patients, including $71 \%$ treated via MTS and $81 \%$ treated via ETS, with a remarkable $70 \%$ of ETS cases in remission off all medical therapy. Rates of hormone control were similar in the ETS and MTS groups at last follow-up ( $\mathrm{p}=0.34$; Table 6).

Preoperative tumor volumes averaged $1.7 \mathrm{~cm}^{3}$ for $\mathrm{GH}-$ 
TABLE 4. Radiographic outcomes

\begin{tabular}{|c|c|c|c|}
\hline Outcome & $\begin{array}{l}\text { Total Cohort, } \\
n=212^{*}\end{array}$ & $\begin{array}{c}\text { Microscopic Only, } \\
n=130\end{array}$ & $\begin{array}{l}\text { Endoscopic Only, } \\
\qquad n=75\end{array}$ \\
\hline \multicolumn{4}{|l|}{ Intraop MRI read } \\
\hline GTR & $64(30)$ & $39(30)$ & $24(32)$ \\
\hline NTR & $99(47)$ & $56(43)$ & $40(53)$ \\
\hline STR & $49(23)$ & $35(27)$ & $11(15)$ \\
\hline Further resection after MRI & $131(62)$ & $79(61)$ & $47(63)$ \\
\hline \multicolumn{4}{|l|}{ Initial postop MRI read† } \\
\hline GTR & $105(53)$ & $54(44)$ & $50(69)$ \\
\hline NTR & $55(28)$ & $40(33)$ & $13(18)$ \\
\hline STR & $39(20)$ & $29(24)$ & $8(11)$ \\
\hline Recurrence/growth & $1(0.5)$ & $0(0)$ & $1(1)$ \\
\hline \multicolumn{4}{|l|}{ Final postop MRI read $\ddagger$} \\
\hline GTR & $111(56)$ & $58(47)$ & $52(72)$ \\
\hline NTR/small residual & $37(19)$ & $24(20)$ & $11(15)$ \\
\hline Stable residual & $41(21)$ & $32(26)$ & $7(10)$ \\
\hline Recurrence/growth & $11(6)$ & $9(7)$ & $2(3)$ \\
\hline Radiographic progression† & $29(15)$ & $24(20)$ & $5(7)$ \\
\hline Further treatment of NFA§ & $18(13)$ & $14(16)$ & $4(8)$ \\
\hline RT & $8(6)$ & $5(6)$ & $3(6)$ \\
\hline Surgery & $8(6)$ & $8(9)$ & $0(0)$ \\
\hline Surgery + RT & $2(1)$ & $1(1)$ & $1(2)$ \\
\hline Median $(95 \% \mathrm{Cl})$ radiographic $\mathrm{PFS}$ in mos & $\begin{array}{c}107.4 \text { (98.96-no } \\
\text { upper limit) }\end{array}$ & $\begin{array}{c}107.4 \text { (98.96-no } \\
\text { upper limit) }\end{array}$ & Not reached \\
\hline
\end{tabular}

$\mathrm{RT}=$ radiation treatment.

Radiographic outcomes are evaluated based on surgical approach. EOR was defined as GTR, NTR, or STR. Initial postoperative MRI results are further classified as recurrence/growth if there was an increase in size following surgery. Late postoperative MRI studies were evaluated based on categories of persistent GTR, NTR, stable residual, or recurrence/growth. EOR was significantly higher in the ETS group compared with the MTS group $(p<0.001)$ and was significantly increased with iMRI across treatment groups $(p=0.0001)$. Only $8 \%$ of NFAs treated endoscopically required further treatment for residual or recurrence, including 1 case $(2 \%)$ requiring further surgery. Unless otherwise indicated, values are expressed as the number of patients (\%).

* This includes 7 patients treated via a combined MTS and ETS approach.

† This includes 200 total patients (123 microscopic only, 72 endoscopic only).

$\ddagger$ This includes 200 total patients at last follow-up.

$\S$ This includes 138 total NFAs with follow-up $\geq 30$ days (86 microscopic only, 48 endoscopic only).

secreting, $1.21 \mathrm{~cm}^{3}$ for ACTH-secreting, and $4.16 \mathrm{~cm}^{3}$ for PRL-secreting tumors. A statistical difference in tumor volumes between the remission and nonremission groups was not reached $\left(1.36 \mathrm{~cm}^{3}\right.$ vs $\left.4.09 \mathrm{~cm}^{3} ; \mathrm{p}=0.21\right)$.

Preoperative hormone deficits were improved or resolved in 29/64 (45\%) cases, including 32\% of MTS and $65 \%$ of ETS patients. ETS resulted in significantly higher hormone deficit improvement than MTS $(p=0.01)$. New anterior pituitary hormone deficits were seen in $6 \%$ of all patients. Permanent diabetes insipidus (DI) was observed in only $6 / 212(3 \%)$ cases, including $2 \%$ of MTS and $5 \%$ of ETS cases (Tables 6 and 7).

\section{Visual Outcomes}

Visual deficits improved in 80/81 (99\%) patients with follow-up evaluations, including $100 \%$ of cases treated via ETS. There were no cases of new VF deficits following surgery.

\section{Complications}

Serious complications requiring reoperation or resulting in death occurred in 3 cases (1.4\%), including 1 case of carotid artery injury via combined MTS/ETS (1/212, $0.5 \%), 1$ case of postoperative hematoma via MTS (1/130, $0.8 \%$ ), and 1 case of a CSF leak requiring reoperation via ETS $(1 / 75,1.3 \%)$. The carotid injury and CSF leak in these cases occurred prior to iMRI, and there was no direct causation between further resection and postoperative hematoma in the third case. Minor postoperative complications (headache, transient hyponatremia, spinal headaches, CSF leak that resolved with lumbar drainage, asymptomatic deep venous thrombosis, or unrelated medical complication requiring readmission) occurred in 22/212 (10\%) patients (Table 7). There were no cases of postoperative infection or worsening visual function following surgery.

Intraoperative CSF leaks occurred in 76 cases (36\%), including $33 \%$ of MTS and $40 \%$ of ETS cases. Postop- 
Juthani et al.
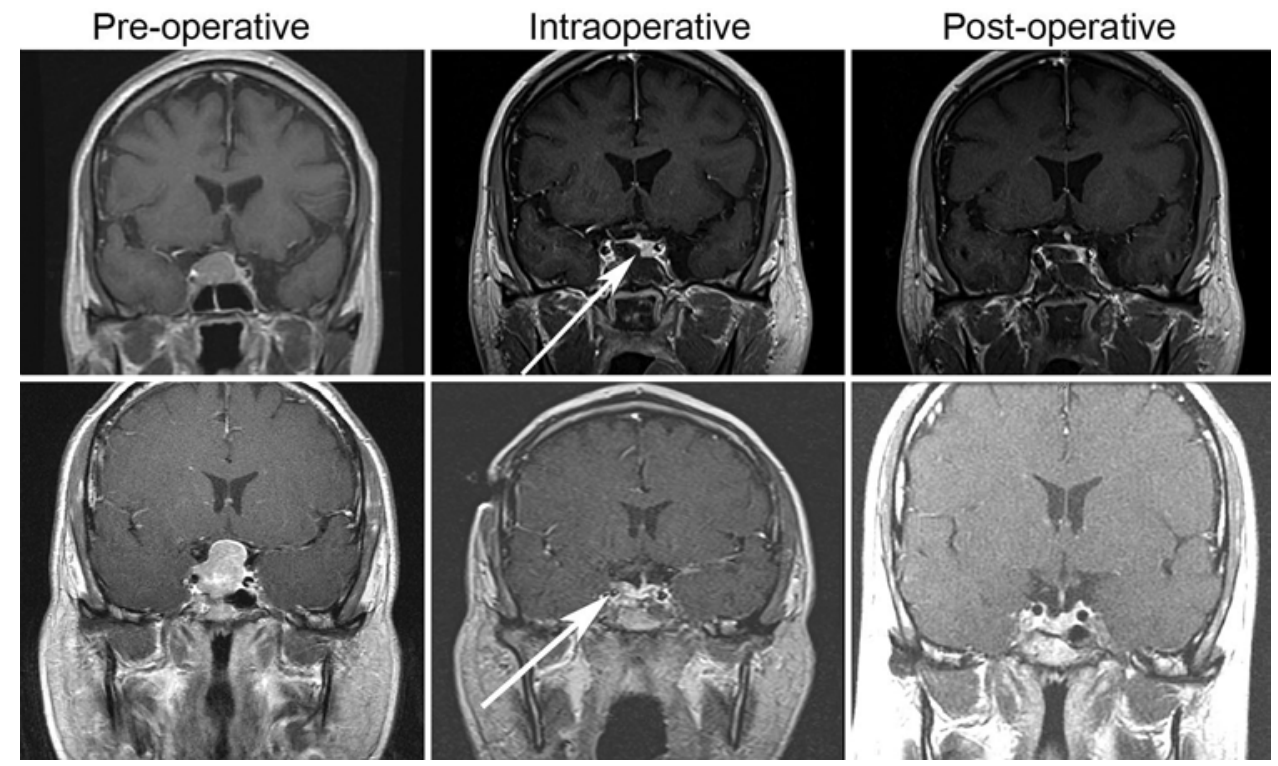

FIG. 1. Representative cases showing decision-making and outcomes based on iMRI. Upper: Coronal MR images demonstrate intraoperative evidence for residual tumor nested within normal gland tissue on iMRI (arrow). The residual was not evident intraoperatively and the surgeon elected not to explore the area because there was very little normal gland tissue. However, after iMRI the area was explored and the residual tumor removed, as shown on the early postoperative image. Lower: Coronal MR images demonstrate residual tumor (arrow) in the superior aspect of the right cavernous sinus that was not seen intraoperatively, despite exploration with the endoscope. The tumor was resected following iMRI, as shown in the postoperative scan.

erative leaks occurred in $1.4 \%$ (3/212 cases), and the rate was similar in MTS and ETS (Table 6). Of the 3 cases with postoperative leaks, only 1 required surgical repair as detailed above.

There were no complications directly attributed to further resection after iMRI.

\section{Discussion}

The use of iMRI during pituitary adenoma resection prompted further resection in $62 \%$ of cases, resulting in significantly higher rates of GTR and NTR. The low rates of GTR on iMRI are reflective of an inherent bias in all iMRI studies due to a tendency to stop the initial resection earlier than in those performed without iMRI. This limits attributing GTR rates directly to the use of iMRI but is reflective of the fact that stopping prior to more aggressive resection may allow surgeons to complete the resection more safely guided by iMRI. These findings were signifi-

TABLE 5. Radiographic outcomes based on cavernous sinus invasion by Knosp grading

\begin{tabular}{cccc}
\hline \multicolumn{1}{c}{ Outcome } & $\begin{array}{c}\text { Total Cohort, } \\
\mathrm{n}=212^{*}\end{array}$ & $\begin{array}{c}\text { Microscopic Only, } \\
\mathrm{n}=130\end{array}$ & $\begin{array}{c}\text { Endoscopic Only, } \\
\mathrm{n}=75\end{array}$ \\
\hline Knosp $\geq 3$ CSI & $24(11)$ & $14(11)$ & $7(9)$ \\
\hline iMRI GTR & $2(8)$ & $1(7)$ & $1(14)$ \\
\hline Further resection after iMRI & $15(63)$ & $8(57)$ & $5(71)$ \\
\hline Initial postop MRI GTR & $1(4)$ & $0(0)$ & $31(41)$ \\
\hline Knosp <3 CSI & $86(41)$ & $52(40)$ & $9(29)$ \\
\hline iMRI GTR & $14(16)$ & $4(8)$ & $21(70)$ \\
\hline Initial postop MRI GTR & $33(41)$ & $11(22)$ & $37(49)$ \\
\hline No CSI & $102(48)$ & $64(49)$ & $14(38)$ \\
\hline iMRI GTR & $48(47)$ & $34(53)$ & $28(80)$ \\
\hline Initial postop MRI GTR $\ddagger$ & $71(74)$ & $43(72)$ & \\
\hline
\end{tabular}

Radiographic outcomes are evaluated based on surgical approach and stratified based on Knosp $<3$ and Knosp $\geq 3$

CSI. Use of iMRI significantly increased GTR rates in patients with Knosp $<3$ CSI $(p<0.0001)$ but not in patients with Knosp $\geq 3 \mathrm{CSI}$.

* This includes 7 patients treated via a combined MTS and ETS approach.

† Reflects total excluding 5 patients who did not receive MRI within 6 months, including 3 microscopic, 1 endoscopic, and 1 with both approaches.

$\ddagger$ Reflects total excluding 6 patients who did not receive MRI with 6 months, including 4 microscopic and 2 endoscopic. 

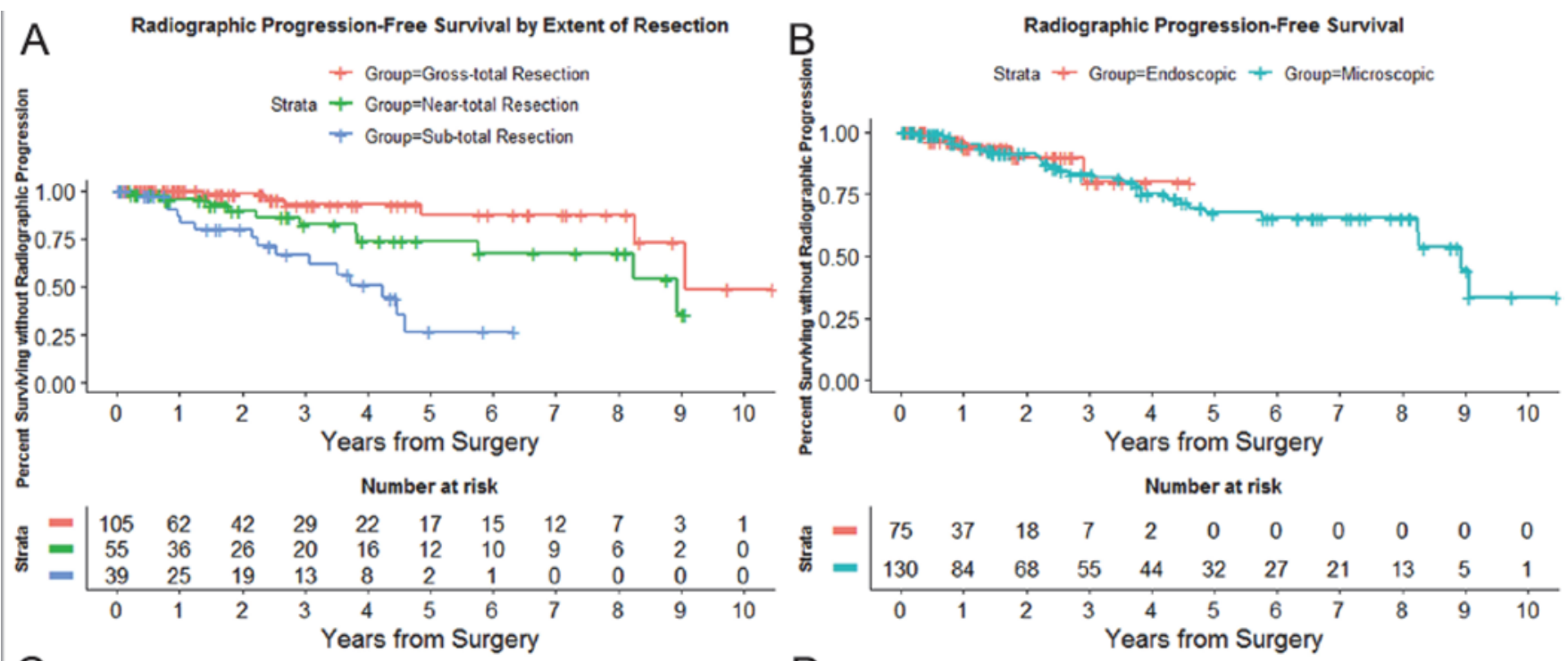

C

Radiographic Progression-Free Survival
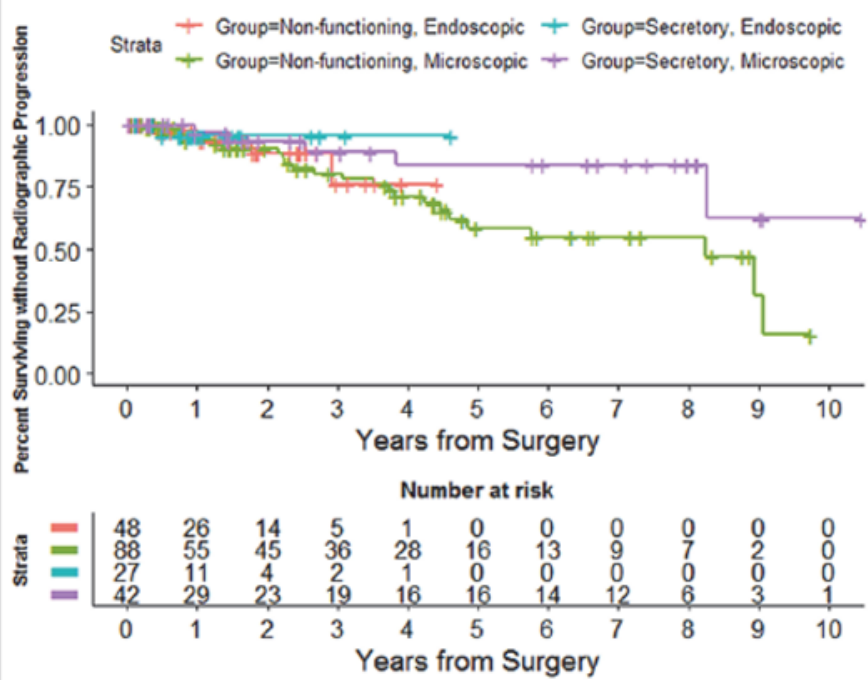

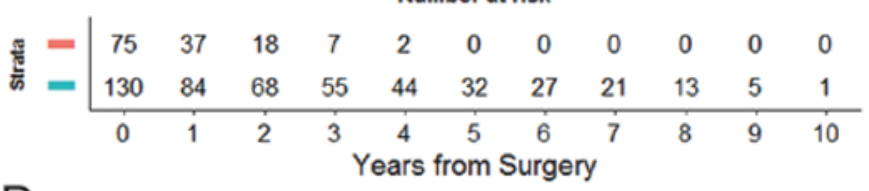

Recurrence-Free Survival in Secretory Adenomas

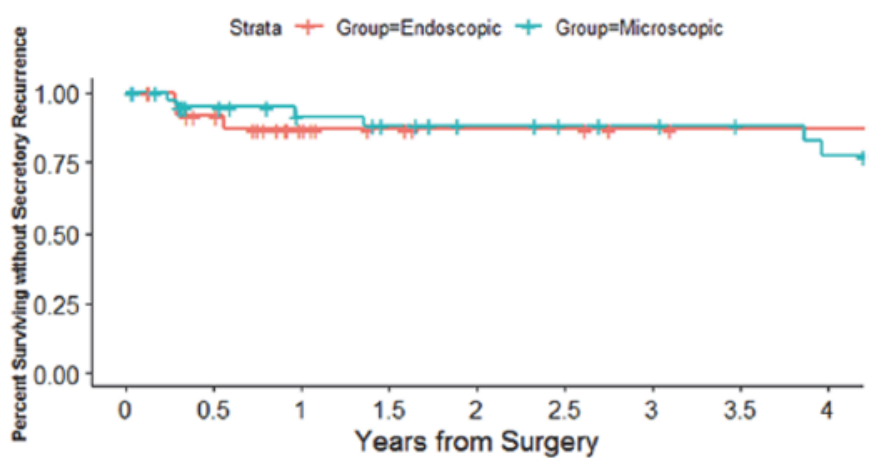

$\frac{⿱ 8}{8}=$\begin{tabular}{ccccccccc}
27 & 20 & 10 & 6 & 4 & 4 & 2 & 1 & 1 \\
42 & 32 & 27 & 24 & 21 & 19 & 18 & 16 & 14 \\
\hline 0 & 0.5 & 1 & 1.5 & 2 & 2.5 & 3 & 3.5 & 4 \\
Years from Surgery & & &
\end{tabular}

FIG. 2. Survival analysis. PFS was evaluated based on both radiographic and clinical endocrine measures. A: Radiographic PFS by iMRI read, stratified by EOR. Increase in EOR was significantly associated with increased PFS. B: Radiographic PFS by endoscopic and microscopic approaches. C: Radiographic PFS in NFAs and in secretory adenomas. D: RFS in secretory adenomas.

cant in both the ETS and MTS groups. As expected, use of iMRI did not improve GTR in cases of Knosp $\geq 3$ CSI but did result in further resection and GTR rates in Knosp 1 and 2 CSI. This was attributed to the inherent inability to resect residual tumor lateral to the carotid via a transsphenoidal approach, making the visualization on MRI irrelevant to GTR rates for these tumors. The low-grade CSI subgroup highlighted the improvement in outcomes with the endoscope (70\% GTR) compared with the microscope (22\% GTR), consistent with expectations. This suggests that for macroadenomas with cavernous sinus "extension" medial to the carotid, iMRI may inform further endoscopic resection, which may not be possible with the more limited microscope view regardless of identification on iMRI.

This series was concordant with others that have reported significantly higher rates of GTR and hormone remission when using the endoscope compared with the microscope.
However, rates of GTR noted on iMRI were not significantly different between the groups, suggesting that iMRI resulted in a greater benefit in the endoscopic compared with the microscopic cases, countering suggestions that the endoscope obviates the need for intraoperative imaging. Interestingly, the recent prospective Transsphenoidal Extent of Resection (TRANSSPHER) trial comparing outcomes for NFAs treated endoscopically versus microscopically demonstrated similar EORs between the groups; however, the authors noted that the experience of the surgeons was significantly higher in the MTS group ${ }^{4}$ which may account for the lack of perceived benefit. Additionally, in this series, direct comparison of long-term tumor control and hormone remission between ETS and MTS groups is limited by the longer follow-up time in the MTS group.

Whereas several of the studies listed previously have evaluated the use of iMRI, our study is unique in high- 
TABLE 6. Clinical and secretory hormone outcomes

\begin{tabular}{|c|c|c|c|}
\hline Outcome & $\begin{array}{c}\text { Total Cohort, } \\
n=212^{*}\end{array}$ & $\begin{array}{c}\text { Microscopic Only, } \\
n=130\end{array}$ & $\begin{array}{l}\text { Endoscopic Only, } \\
\qquad n=75\end{array}$ \\
\hline VF deficit & 81 & 53 & 25 \\
\hline Improved/resolved & $80(99)$ & $52(98)$ & $25(100)$ \\
\hline Stable & $1(1)$ & $1(2)$ & $0(0)$ \\
\hline Secretory adenoma & $72(34)$ & $42(32)$ & $27(36)$ \\
\hline Initial hormone remission† & $46 / 72(64)$ & $23 / 42(55)$ & $22 / 27(81)$ \\
\hline $\mathrm{GH}$ & $19 / 29(66)$ & $10 / 17(59)$ & $9 / 11(82)$ \\
\hline ACTH & $16 / 20(80)$ & $7 / 9(78)$ & $8 / 10(80)$ \\
\hline PRL & $11 / 23(48)$ & $6 / 16(38)$ & $5 / 6(83)$ \\
\hline Recurrence after remission & $11 / 46(24)$ & $7 / 23(30)$ & $4 / 22(18)$ \\
\hline Median $(95 \% \mathrm{Cl})$ RFS in mos & $81.6(47.6-100.2)$ & $81.6(47.6-100.2)$ & Not reached \\
\hline Further treatment of secretory adenomał & $35(49)$ & $24(57)$ & $9(33)$ \\
\hline Medical only & $23(32)$ & $16(38)$ & $6(22)$ \\
\hline RT & $2(3)$ & $0(0)$ & $2(7)$ \\
\hline Med + RT & $4(6)$ & $3(7)$ & $1(4)$ \\
\hline Surgery & $0(0)$ & $0(0)$ & 0 \\
\hline Med + surgery & $5(7)$ & $4(10)$ & 0 \\
\hline Med + surgery + RT & $1(1)$ & $1(2)$ & 0 \\
\hline Hormone remission at last FU & $54(75)$ & $30(71)$ & $22(81)$ \\
\hline Hormone remission at last FU off medication $\ddagger$ & $43(60)$ & $23(55)$ & $19(70)$ \\
\hline \multicolumn{4}{|l|}{ Preop hormone deficit improved/resolved } \\
\hline None & $35 / 64(55)$ & $23 / 34(68)$ & $9 / 26(35)$ \\
\hline 1 or more & $29 / 64(45)$ & $11 / 34(32)$ & $17 / 26(65)$ \\
\hline \multicolumn{4}{|l|}{ Preop deficit improvement by type } \\
\hline Hypocortisolemia & $7 / 23(30)$ & $4 / 15(27)$ & $3 / 7(43)$ \\
\hline Hypogonadism & $20 / 50(40)$ & $6 / 27(22)$ & $8 / 12(67)$ \\
\hline Hypothyroidism & $14 / 31(45)$ & $6 / 18(33)$ & $13 / 20(65)$ \\
\hline GH deficiency & $2 / 5(40)$ & $1 / 4(25)$ & $1 / 1(100)$ \\
\hline
\end{tabular}

Clinical outcomes were evaluated based on treatment group. VF deficits improved or resolved in $99 \%$ of patients, including $100 \%$ of patients treated via ETS. Hormone remission was defined as return to normal hormone levels off of medical therapy. No patients treated via ETS required further surgery for secretory tumor hormone secretion. ETS resulted in higher rates of hormone remission than MTS $(p=0.02)$. Preoperative hormone deficits were evaluated for lack of improvement or resolution of $\geq 1$ preoperative deficit. Preoperative deficits were improved in $65 \%$ of patients treated via ETS. Unless otherwise indicated, values are expressed as the number of patients (\%).

* This includes 7 patients treated via a combined MTS and ETS approach.

$\dagger$ Hormone remission defined off medical therapy, without any additional treatment.

$\ddagger$ Hormone remission on or off medication, total cohort 72 secretory tumors.

lighting improvement in hormone outcomes. Although the study reestablished higher rates of hormone remission in tumors treated via ETS compared with MTS, differences in outcomes were most notable in GH- and PRLsecreting tumors, which are typically macroadenomas, compared with corticotropin-producing tumors, which tend to be microadenomas. Indeed, in a subgroup analysis of ACTH-secreting adenomas, there was no difference in cure rates between the microscopic (78\%) and endoscopic $(80 \%)$ approaches. More notably, however, the use of iMRI with ETS results in a markedly high rate of hormone remission (81\%) off medication, with only an $18 \%$ rate of recurrence; of these recurring cases, none required further surgery, and $81 \%$ had controlled hormone secretion at last follow-up.
General hormone remission rates have been reported at approximately $62.5 \%$ even with use of the endoscope and iMRI,${ }^{17}$ but vary based on hormone type and tumor size. ${ }^{24}$ Remission rates for ACTH-secreting tumors have been reported at $65 \%-87 \%$ with the microscope and $72 \%-95 \%$ with the endoscope; ${ }^{25,26}$ for GH-secreting tumors at $30 \%-$ $70 \%$ with the microscope and $46 \%-70 \%$ with the endoscope; ${ }^{26,27}$ and with lower rates for PRL-secreting tumors at $66 \%$ with the microscope and $42 \%-76 \%$ with the endoscope. ${ }^{26,28}$ In comparison, whereas our remission rates were comparable for microscopic cases, we report very high remission rates in secretory tumors treated endoscopically across hormone type (80\%-83\%), with the difference most marked for GH- and PRL-secreting tumors. These results suggest a potential benefit for hormone and 
TABLE 7. Complications in 212 patients with pituitary adenoma

\begin{tabular}{|c|c|c|c|}
\hline Complication & $\begin{array}{c}\text { Total Cohort, } \\
n=212^{*}\end{array}$ & $\begin{array}{l}\text { Microscopic Only, } \\
n=130\end{array}$ & $\begin{array}{l}\text { Endoscopic Only, } \\
\qquad n=75\end{array}$ \\
\hline \multicolumn{4}{|l|}{ New hormone deficits } \\
\hline Transient DI & $36 / 212(17)$ & $23 / 130(18)$ & $12 / 75(16)$ \\
\hline Permanent DI & $6 / 212(3)$ & $2 / 130(2)$ & $4 / 75(5)$ \\
\hline Other & $13 / 212(6)$ & $8 / 130(6)$ & $5 / 75(7)$ \\
\hline Permanent DI or other & $17 / 212(8)$ & $9 / 130(7)$ & $8 / 75(11)$ \\
\hline \multicolumn{4}{|l|}{ Nonhormone/nonleak complications } \\
\hline Serious postop complication requiring reop & $3 / 212(1)$ & $1 / 130(0.8)$ & $1 / 75(1)$ \\
\hline Minor postop complication & $22 / 212(10)$ & $10 / 130(8)$ & $12 / 75(16)$ \\
\hline Intraop CSF leak & $76 / 212(36)$ & $43 / 130(33)$ & $30 / 75(40)$ \\
\hline \multicolumn{4}{|l|}{ Secretory repair type $\dagger$} \\
\hline Gelfoam & $19(25)$ & $16(37)$ & $3(10)$ \\
\hline Mucosa or fat graft \pm Gelfoam & $22(29)$ & $19(44)$ & $3(10)$ \\
\hline Dural synthetic; Alloderm/Duragen & $13(17)$ & $8(19)$ & $4(13)$ \\
\hline NS flap & $8(11)$ & $0(0)$ & $8(27)$ \\
\hline NS flap + dural synthetic & $8(11)$ & $0(0)$ & $7(23)$ \\
\hline NS flap + dural synthetic + fat graft & $6(8)$ & $0(0)$ & $5(17)$ \\
\hline Lumbar drain & $27(13)$ & $24(18)$ & $3(4)$ \\
\hline Postop leak & $3 / 212(1)$ & $2 / 130(2)$ & $1 / 75(1)$ \\
\hline Resolved w/ lumbar drain & $2 / 3(66)$ & $2 / 2(100)$ & $0 / 1(0)$ \\
\hline Surgical repair & $1 / 3(33)$ & $0 / 2(0)$ & $1 / 1(100)$ \\
\hline \multicolumn{4}{|c|}{$\begin{array}{l}\text { NS = nasoseptal. } \\
\text { Postoperative complications were evaluated for new hormone deficit, CSF leak, and medical or surgical complications. } \\
\text { Medical and surgical complications were stratified as minor (headache, transient hyponatremia, spinal headaches, CSF } \\
\text { leak that resolved with lumbar drainage, asymptomatic deep venous thrombosis, or unrelated medical complication } \\
\text { requiring admission) or major (requiring reoperation or leading to death). The rate of permanent hormone deficit includ- } \\
\text { ing DI was } 8 \% \text {, with a low rate of postoperative CSF leak at } 1 \% \text {, serious complications at } 1 \% \text {, and minor complications } \\
\text { at } 11 \% \text {. } \\
\text { * This includes } 7 \text { patients treated via a combined MTS and ETS approach. } \\
\dagger \text { All categories are with or without dural sealant. }\end{array}$} \\
\hline
\end{tabular}

radiographic outcomes for secretory tumors treated via ETS. Tumor volumes were higher in the "no remission" group, but differences did not reach statistical significance $\left(4.09 \mathrm{~cm}^{3}\right.$ vs $\left.1.36 \mathrm{~cm}^{3}\right)$. The median secretory RFS in this cohort was 81.6 months, demonstrating durable hormone control following iMRI-guided resection and emphasizing the importance of long-term follow-up.

Although use of iMRI raises the concern of encouraging overly aggressive resection leading to deficit, our results suggest a potential protective effect in the case of pituitary adenomas. Here we report an overall rate of new hormone deficit of only $8 \%$ excluding transient DI, which compares favorably to rates of $8.75 \%-26 \%$ in other series (Supplemental Table 1). We also report improvement in preoperative deficits in $65 \%$ of patients treated via ETS, compared with $7.4 \%-55 \%$ reported in the literature, as summarized in Supplemental Table 1. These differences could be in part related to our systematic efforts to preserve normal tissue and consistent reliance on intraoperative imaging to identify the normal gland and stalk. In the absence of iMRI, ambiguity in these structures may lead to partial gland resection or increased manipulation to maximize resection, which may explain the seemingly protective effect of iMRI. Laws et al.preoperatively evaluated pituitary adenomas with high-resolution MRI to systematically identify the location of the normal gland and subsequently reported particularly low rates of new postoperative hormone deficits of $8.75 \%$, excluding syndrome of inappropriate antidiuretic hormone; however, only $7.4 \%$ of patients saw improvement in preoperative deficits. 32 Our results highlight the utility of iMRI in the preservation of pituitary function and augmentation of recovery from preoperative hormone deficit. In our series, slightly higher rates of improvement were seen in preoperative hypothyroidism (45\%) compared with hypocortisolemia (30\%), although other studies have suggested that adrenal insufficiency is the most likely to recover. ${ }^{29,30}$ However, there is marked variability in reports of postoperative hormone recovery, particularly with regard to the cortisol axis. ${ }^{31}$ Nonhormone complications were rare, with serious complications in $1.4 \%$ of patients and CSF leaks in $1 \%$ of patients, of which only 1 patient $(0.4 \%)$ required reoperation. There were no cases of postoperative infection or meningitis, and there were no complications directly attributed to further resec- 
tion after iMRI. These results compare favorably with the published literature, where the rate of postoperative CSF leaks ranges from $1 \%$ to $6 \%{ }^{26}$ and other serious complications are rare, with no evidence of increase in complication rate compared to series without use of iMRI.,26,27 Overall, our findings support the general advantages of the endoscopic over the microscopic approach and augmented results with iMRI.

iMRI inevitably adds to the operative time, estimated to be approximately $45-60$ minutes, although evolving workflows have reduced time requirements, and iMRI often eliminates the need for postoperative MRI prior to discharge. Future work evaluating the cost-effectiveness of performing iMRI in these cases would be useful in determining its value.

Taken together, this study demonstrates that the use of iMRI results in high rates of GTR correlated with increased PFS, high rates of hormone remission, and improvement in pituitary hormone function, with low rates of hormone deficits and complications. The data also suggest that integration of iMRI into the surgical workflow can result in protection of pituitary gland function. These results may be most pronounced when paired with the use of the endoscope for resecting macroadenomas with Knosp 1 and 2 CSI and for secretory tumors.

\section{Study Limitations}

There are several limitations to the current study. The duration of follow-up in microscopic and endoscopic groups is inherently longer in the former, due to the fact that the endoscope was introduced at our institution only in 2014 , prior to which all cases were done microscopically. Timing of iMRI has a subjective component: although it is often done after maximal resection, it is sometimes done as a check on anatomy, overestimating the value of iMRI and limiting meaningful comparison of GTRs before and after iMRI. This study, however, reflects the experience of a single neurosurgeon and ENT team, minimizing some variability in the "threshold" for MRI. Despite these limitations, we believe that the data provide valuable insight into the potential benefits of iMRI in increasing resection and preserving and restoring hormone function during resection of pituitary adenomas.

\section{Conclusions}

The data suggest that iMRI is a safe and effective method to increase postoperative EOR in pituitary adenomas when combined with the endoscope. Use of iMRI resulted in high rates of radiographic PFS for NFAs and high rates of hormone remission for secretory tumors compared with rates reported in the literature, particularly when iMRI was paired with the endoscope. These results were achieved with low rates of complications and new endocrinopathy, as well as improvement of preoperative hormone deficits in a majority of cases. This study supports the use of iMRI to tailor more aggressive tumor resection while preserving pituitary hormone function due to early gland identification. The use of iMRI for resecting pituitary adenomas may be recommended when available and is advisable particularly in the case of hormone-secreting tumors, tumors with grade 1 and 2 cavernous sinus involvement, and possibly giant adenomas. Prospective evaluation of the role of iMRI coupled with a cost-effectiveness analysis should be pursued.

\section{Acknowledgments}

This work was supported by NIH P30 CA 008748.

\section{References}

1. Fang J, Xie S, Li N, Jiang Z. Postoperative complications of endoscopic versus microscopic transsphenoidal pituitary surgery: a meta-analysis. J Coll Physicians Surg Pak. 2018;28(7):554-559.

2. Schaberg MR, Anand VK, Schwartz TH, Cobb W. Microscopic versus endoscopic transnasal pituitary surgery. Curr Opin Otolaryngol Head Neck Surg. 2010;18(1):8-14.

3. Singh H, Essayed WI, Cohen-Gadol A, et al. Resection of pituitary tumors: endoscopic versus microscopic. J Neurooncol. 2016;130(2):309-317.

4. Little AS, Kelly DF, White WL, et al. Results of a prospective multicenter controlled study comparing surgical outcomes of microscopic versus fully endoscopic transsphenoidal surgery for nonfunctioning pituitary adenomas: the Transsphenoidal Extent of Resection (TRANSSPHER) Study. J Neurosurg. 2019;132(4):1043-1053.

5. Esquenazi Y, Essayed WI, Singh H, et al. Endoscopic endonasal versus microscopic transsphenoidal surgery for recurrent and/or residual pituitary adenomas. World Neurosurg. 2017;101:186-195.

6. Sylvester PT, Evans JA, Zipfel GJ, et al. Combined high-field intraoperative magnetic resonance imaging and endoscopy increase extent of resection and progression-free survival for pituitary adenomas. Pituitary. 2015;18(1):72-85.

7. Bakhsheshian J, Wheeler S, Strickland BA, et al. Surgical outcomes following repeat transsphenoidal surgery for nonfunctional pituitary adenomas: a retrospective comparative study. Oper Neurosurg (Hagerstown). 2019;16(2):127-135.

8. Sughrue ME, Chang EF, Gabriel RA, et al. Excess mortality for patients with residual disease following resection of pituitary adenomas. Pituitary. 2011;14(3):276-283.

9. Akbari H, Malek M, Ghorbani M, et al. Clinical outcomes of endoscopic versus microscopic trans-sphenoidal surgery for large pituitary adenoma. Br J Neurosurg. 2018;32(2):206209.

10. Kubben PL, ter Meulen KJ, Schijns OE, et al. Intraoperative MRI-guided resection of glioblastoma multiforme: a systematic review. Lancet Oncol. 2011;12(11):1062-1070.

11. Senft C, Bink A, Franz K, et al. Intraoperative MRI guidance and extent of resection in glioma surgery: a randomised, controlled trial. Lancet Oncol. 2011;12(11):997-1003.

12. Berkmann S, Schlaffer S, Nimsky C, et al. Intraoperative high-field MRI for transsphenoidal reoperations of nonfunctioning pituitary adenoma. J Neurosurg. 2014;121(5):11661175 .

13. Soneru CP, Riley CA, Hoffman K, et al. Intra-operative MRI vs endoscopy in achieving gross total resection of pituitary adenomas: a systematic review. Acta Neurochir (Wien). 2019;161(8):1683-1698.

14. García S, Reyes L, Roldán P, et al. Does low-field intraoperative magnetic resonance improve the results of endoscopic pituitary surgery? Experience of the implementation of a new device in a referral center. World Neurosurg. 2017;102:102110.

15. Hlaváč M, Knoll A, Etzrodt-Walter G, et al. Intraoperative MRI in transsphenoidal resection of invasive pituitary macroadenomas. Neurosurg Rev. 2019;42(3):737-743.

16. Netuka D, Masopust V, Belšán T, et al. One year experience 
with 3.0 T intraoperative MRI in pituitary surgery. Acta Neurochir Suppl. 2011;109:157-159.

17. Pal'a A, Knoll A, Brand C, et al. The value of intraoperative magnetic resonance imaging in endoscopic and microsurgical transsphenoidal pituitary adenoma resection. World Neurosurg. 2017;102:144-150.

18. Serra C, Burkhardt JK, Esposito G, et al. Pituitary surgery and volumetric assessment of extent of resection: a paradigm shift in the use of intraoperative magnetic resonance imaging. Neurosurg Focus. 2016;40(3):E17.

19. Staartjes VE, Serra C, Maldaner N, et al. The Zurich Pituitary Score predicts utility of intraoperative high-field magnetic resonance imaging in transsphenoidal pituitary adenoma surgery. Acta Neurochir (Wien). 2019;161(10):2107-2115.

20. Zaidi HA, De Los Reyes K, Barkhoudarian G, et al. The utility of high-resolution intraoperative MRI in endoscopic transsphenoidal surgery for pituitary macroadenomas: early experience in the Advanced Multimodality Image Guided Operating suite. Neurosurg Focus. 2016;40(3):E18.

21. Zhang H, Wang F, Zhou T, et al. Analysis of 137 patients who underwent endoscopic transsphenoidal pituitary adenoma resection under high-field intraoperative magnetic resonance imaging navigation. World Neurosurg. 2017;104:802-815.

22. Zhang Z, Yang K, Xia Y, et al. High-field intraoperative magnetic resonance imaging increases extent of resection and progression-free survival for nonfunctioning pituitary adenomas. World Neurosurg. 2019;127:e925-e931.

23. Netuka D, Májovský M, Masopust V, et al. Intraoperative magnetic resonance imaging during endoscopic transsphenoidal surgery of growth hormone-secreting pituitary adenomas. World Neurosurg. 2016;91:490-496.

24. Elshazly K, Kshettry VR, Farrell CJ, et al. Clinical outcomes after endoscopic endonasal resection of giant pituitary adenomas. World Neurosurg. 2018;114:e447-e456.

25. Broersen LHA, van Haalen FM, Biermasz NR, et al. Microscopic versus endoscopic transsphenoidal surgery in the Leiden cohort treated for Cushing's disease: surgical outcome, mortality, and complications. Orphanet J Rare Dis. 2019;14(1):64.

26. Dallapiazza RF, Jane JA Jr. Outcomes of endoscopic transsphenoidal pituitary surgery. Endocrinol Metab Clin North Am. 2015;44(1):105-115.

27. Chen CJ, Ironside N, Pomeraniec IJ, et al. Microsurgical versus endoscopic transsphenoidal resection for acromegaly: a systematic review of outcomes and complications. Acta Neurochir (Wien). 2017;159(11):2193-2207.

28. Akin S, Isikay I, Soylemezoglu F, et al. Reasons and results of endoscopic surgery for prolactinomas: 142 surgical cases. Acta Neurochir(Wien). 2016;158(5):933-942.

29. Harary M, DiRisio AC, Dawood HY, et al. Endocrine function and gland volume after endoscopic transsphenoidal surgery for nonfunctional pituitary macroadenomas. J Neurosurg. 2018;131(4):1142-1151.

30. Kim JH, Lee JH, Lee JH, et al. Endoscopic transsphenoidal surgery outcomes in 331 nonfunctioning pituitary adenoma cases after a single surgeon learning curve. World Neurosurg. 2018;109:e409-e416.
31. Jahangiri A, Wagner JR, Han SW, et al. Improved versus worsened endocrine function after transsphenoidal surgery for nonfunctional pituitary adenomas: rate, time course, and radiological analysis. J Neurosurg. 2016;124(3):589-595.

32. Laws ER Jr, Iuliano SL, Cote DJ, et al. A benchmark for preservation of normal pituitary function after endoscopic transsphenoidal surgery for pituitary macroadenomas. World Neurosurg. 2016;91:371-375.

33. Knosp E, Steiner E, Kitz K, Matula C. Pituitary adenomas with invasion of the cavernous sinus space: a magnetic resonance imaging classification compared with surgical findings. Neurosurgery 1993;33(4):610-618.

34. Berkman S, Schlaffer S, Nimsky C, et al. Follow-up and long-term outcome of nonfunctioning pituitary adenoma operated by transsphenoidal surgery with intraoperative highfield magnetic resonance imaging. Acta Neurochir (Wien). 2014;156(12):2233-2243.

\section{Disclosures}

Dr. Geer received clinical or research support for the study described (includes equipment or material) from Novartis, IONIS, Chiasma, Strongbridge, and Corcept. Dr. Tabar is a consultant for BlueRock Therapeutics.

\section{Author Contributions}

Conception and design: Juthani. Acquisition of data: Patel, Cowan, Roguski, Geer, Karimi, Cohen. Analysis and interpretation of data: Tabar, Juthani, Reiner, Roguski, Panageas. Drafting the article: Juthani. Critically revising the article: Tabar. Reviewed submitted version of manuscript: Tabar, Juthani, Reiner, Roguski, Panageas, Geer, Karimi, Cohen. Approved the final version of the manuscript on behalf of all authors: Tabar. Statistical analysis: Tabar, Reiner, Panageas. Administrative/technical/material support: Cowan. Study supervision: Tabar, Panageas.

\section{Supplemental Information}

\section{Online-Only Content}

Supplemental material is available with the online version of the article.

Supplemental Table 1. https://thejns.org/doi/suppl/10.3171/ 2020.4.JNS20178.

\section{Previous Presentations}

Partial data were briefly presented orally at the North American Skull Base Society Annual Meeting in Orlando, Florida, on February $16,2019$.

\section{Correspondence}

Viviane Tabar: Memorial Sloan Kettering Cancer Center, New York, NY.tabarv@mskcc.org; tabarv@mskneurosurgery. 